\title{
Low Frequency Vibration Energy Harvester Using Stopper-Engaged Dynamic Magnifier for Increased Power and Wide Bandwidth
}

\author{
Miah Abdul Halim*, Dae Heum Kim ${ }^{\dagger}$ and Jae Yeong Park*
}

\begin{abstract}
We present a piezoelectric energy harvester with stopper-engaged dynamic magnifier which is capable of significantly increasing the operating bandwidth and the energy (power) harvested from a broad range of low frequency vibrations $(<30 \mathrm{~Hz})$. It uses a mass-loaded polymer beam (primary spring-mass system) that works as a dynamic magnifier for another mass-loaded piezoelectric beam (secondary spring-mass system) clamped on primary mass, constituting a two-degree-of-freedom (2-DOF) system. Use of polymer (polycarbonate) as the primary beam allows the harvester not only to respond to low frequency vibrations but also generates high impulsive force while the primary mass engages the base stopper. Upon excitation, the dynamic magnifier causes mechanical impact on the base stopper and transfers a secondary shock (in the form of impulsive force) to the energy harvesting element resulting in an increased strain in it and triggers nonlinear frequency up-conversion mechanism. Therefore, it generates almost four times larger average power and exhibits over $250 \%$ wider half-power bandwidth than those of its conventional 2-DOF counterpart (without stopper). Experimental results indicate that the proposed device is highly applicable to vibration energy harvesting in automobiles.
\end{abstract}

Keywords: 2-DOF system, Base stopper, Dynamic magnifier, Frequency up-conversion, Mechanical impact, Wideband operation

\section{Introduction}

During last few years, extensive efforts have been exerted to develop a wide variety of piezoelectric energy harvesters to scavenge power effectively from ambient vibration for powering low-power-consumption small wireless electronics [1-4], so that they can be operated autonomously and uninterruptedly by eliminating the need for recharging or replacing their external power sources such as batteries. Typically, a vibration energy harvester delivers maximum power to a matched load only if its resonant frequency matches ambient vibration frequency and power flow decreases as the resonant frequency decreases $[5,6]$. But unfortunately, ambient vibrations are of low frequencies with eccentric nature which may drift over time [7]. A conventional piezoelectric vibration energy harvester is designed as a single-degree-of-freedom (SDOF) model in the form of a single mass-loaded cantilever beam (made of either a piezoelectric bimorph or a piezoelectric layer attached to a non-piezoelectric layer) which is efficient at its 1st resonance mode; 2nd and higher resonance modes with comparatively low response levels occur in excessively high frequencies and are generally ignored. This instinctive behavior results in a narrow operating bandwidth making itself inefficient for power

$\dagger$ Corresponding Author: Department of Chemical Engineering, Kwangwoon University, Korea. (kdh@kw.ac.kr)

* Department of Electronic Engineering, Kwangwoon University,

Korea. (jaepark@kw.ac.kr, ahm.aece@hotmail.com)

Received: September 15, 2015; Accepted: December 17, 2015 harvesting from a wide range of low frequency vibrations.

In order to overcome the narrow bandwidth problem, a number of researchers have demonstrated broadband harvester by using a multi-resonant piezoelectric beam by attaching multiple cantilever beams of natural frequencies close to the first resonance of the primary beam $[8,9]$. A device with two piezoelectric beams connected with elastic springs has also been reported that improves the bandwidth by sacrificing the power [10]. A wideband piezoelectric energy harvester using a second piezoelectric beam as a stopper has also been demonstrated [11]. Beside the SDOF systems, some researchers have presented two-degree-offreedom (2-DOF) systems using two-mass cantilever beam [12-14]. Use of an additional spring-mass system between the conventional SDOF mass-loaded piezoelectric cantilever beam and the harvester base, also known as dynamic magnifier, widens the effective bandwidth by decreasing the frequency gap between its 1 st and 2nd resonance modes $[15,16]$. It also increases the harvester's power output by virtue of magnifying (as the name dynamic magnifier implies) the strain experienced by the piezoelectric beam [17]. However, these harvesters cannot be considered as true wideband as the 2nd resonance peak is still considerably smaller and quite far away from the 1st resonance peak, as well as contain a deep valley between two peaks.

Besides the bandwidth issue, higher power generation under low frequency operating condition is another challenge to handle, because the output power of a vibration 
energy harvester is directly proportional to the cube of its resonant frequency [5]. Any wideband energy harvester with low operating frequency range also suffers from it. Mechanical frequency up-conversion mechanism has been proposed by a number of research groups in order to overcome this challenge. It has been done either by mechanical impact [18-20] or by non-mechanical interaction e.g., magnetic attraction/repulsion [21-23].

In this study, we have demonstrated the details of a mechanical impact driven 2-DOF piezoelectric low frequency vibration energy harvester that has recently been proposed by our group [24]. The device consists of a stopper-engaged flexible (polymer) dynamic magnifier in order to enhance the performance, in terms of both output power and operating bandwidth. Use of polymer not only decreases the resonant frequency but also increases the displacement limit in order to enhance dynamic magnification. The dynamic magnifier engages the harvester base stopper by piecewise linear impact during its vibration, even at a wide range of low frequencies; delivers secondary shock and introduces nonlinear frequency up-conversion mechanism to the power generating element which, in turn, increases the output power significantly by increasing the strain and decreasing the source resistance within it.

\section{Harvester Principle and Fabrication}

\subsection{Device structure and its working}

The schematic structure of the proposed energy harvester is shown in Fig. 1. It is consisted of two parts: the dynamic magnifier comprising a flexible primary beam with a primary mass attached to one end constituting primary spring-mass system and, a relatively smaller and

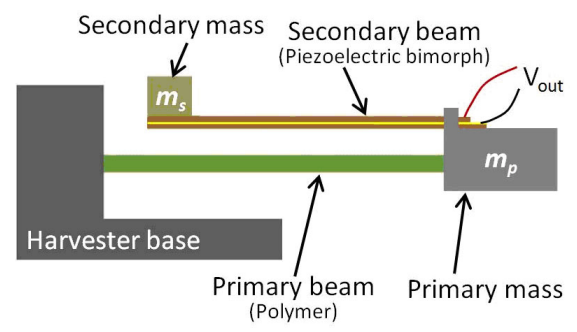

(a)

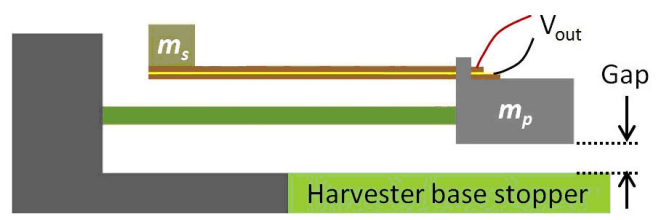

(b)

Fig. 1. Schematic of the proposed energy harvester with dynamic magnifier: (a) conventional 2-DOF structure, and (b) harvester with stopper (HSDM) rigid secondary beam with a small secondary mass attached to one end, other end being clamped to the primary mass in a parallel manner over the primary beam, constituting secondary spring-mass system. The mass ratio is $\mu=5.8$. The overall system is clamped to the anchor that constitutes the conventional 2-DOF system structure. A rigid stopper (also called harvester base stopper) is placed at the bottom of the overall (primary and secondary springmass) vibrating system at such a position that a small gap exists between the fender (lower part) of the primary mass and the base stopper that constitutes the energy harvester with stopper-engaged dynamic magnifier (HSDM). The gap is left small so that the fender can be engaged with the base stopper within its displacement over the applied frequency range.

While low frequency periodic excitation with sufficiently large acceleration is applied, the overall spring-mass system (Primary and secondary) vibrates according to the excitation frequency. The flexible dynamic magnifier allows magnifying the strain experienced by the piezoelectric bimorph beam which, in turns, increase the electrical power output of the harvester. In addition, two close resonances occur which increase the effective operating bandwidth [15]. On the other hand, the maximum displacement of the primary mass (in HSDM) is limited to the threshold value by the base stopper giving rise to non-linearity in the system. The vibration behavior deviates from its normal 2DOF motion and results in a hindrance of the vibration motion, introducing a periodically forced piecewise linear motion (after impact on the stopper) which, in turn, broadens the operating frequency bandwidth. Detailed analysis of the dynamics of this kind of piecewise linear impact can be found in the literature $[25,26]$. This impact mechanism induces a secondary shock to the secondary spring-mass system. As a result, the secondary mass vibrates at its own natural frequency (higher than that of the primary spring-mass system) with exponential decay. The average stress on the piezoelectric layer is increased due to larger displacement of the secondary mass resulting in increased output voltage or power as compared to the conventional energy harvester with dynamic magnifier (EHDM) as shown in Fig. 2.

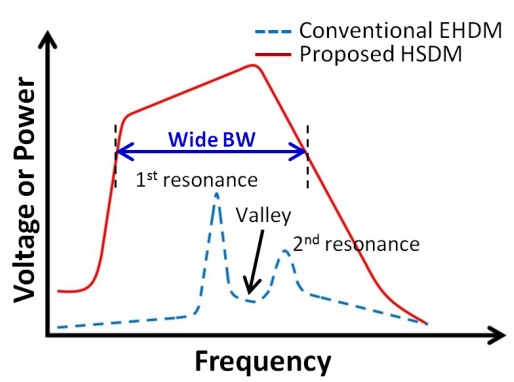

Fig. 2. Expected output characteristics of the proposed HSDM in comparison with its conventional 2-DOF counterpart (EHDM) 


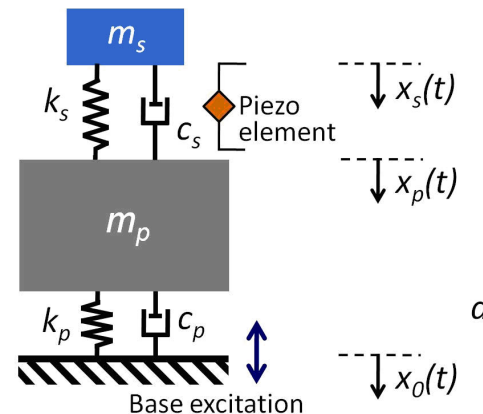

(a)

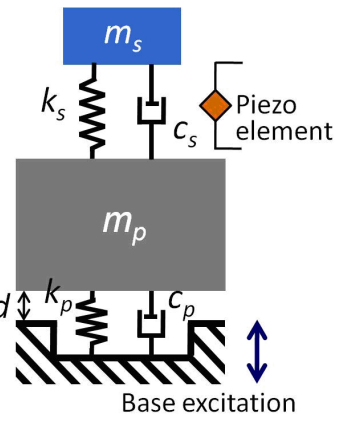

(b)
Fig. 3. Equivalent mechanical model of (a) conventional energy harvester with dynamic magnifier (EHDM), and (b) energy harvester with stopper-engaged dynamic magnifier (HSDM)

\subsection{Electromechanical modeling}

The energy harvester with dynamic magnifier (EHDM) in Fig. 1(a) can be modeled as a conventional 2-DOF model, whereas the energy harvester with stopper-engaged dynamic magnifier (HSDM) in Fig. 1(b) can be modeled as a piecewise linear vibro-impact model for a two-mass cantilevered spring-mass system. The equivalent mechanical models for both EHDM and HSDM are shown in Fig. 3. The dynamic equations of motion of the EHDM can be expressed as [27]

$$
\begin{gathered}
m_{p} \ddot{x}_{p}+c_{p}\left(\dot{x}_{p}-\dot{x}_{0}\right)+k_{p}\left(x_{p}-x_{0}\right)=-m_{s} \ddot{x}_{s} \\
m_{s} \ddot{x}_{s}+c_{s}\left(\dot{x}_{s}-\dot{x}_{p}\right)+k_{s}\left(x_{s}-x_{p}\right)=0
\end{gathered}
$$

where $m_{p}, x_{p}, c_{p}$, and $k_{p}$ are the mass, displacement, damping constant, and stiffness of the primary spring-mass system, respectively; $m_{s}, x_{s}, c_{s}$, and $k_{s}$ are the mass, displacement, damping constant, and stiffness of the secondary spring-mass system, respectively; $x_{0}$ is the displacement of the vibrating base. On the other hand, while low frequency periodic excitation with sufficiently large acceleration $a$ is applied to the HSDM, then in the time interval between two consecutive impacts, its dynamic equations of motion are

$$
\begin{gathered}
m_{p} \ddot{x}_{p}+\left(c_{p}+c_{s}\right) \dot{x}_{p}-c_{s} \dot{x}_{s}+\left(k_{p}+k_{s}\right) x_{p} \\
-k_{s} x_{s}=m_{p} a \sin \omega_{0} t \\
m_{s} \ddot{x}_{s}+c_{s}\left(\dot{x}_{s}-\dot{x}_{p}\right)+k_{s}\left(x_{s}-x_{p}\right)=\delta_{s}(t-\tau)
\end{gathered}
$$

where $\delta_{s}(t-\tau)$ is the impulsive force generated by the secondary shock (while impact occurs) at time $(t-\tau), t>>$. $\tau$ is the time between the start of the cycle (at $t=0$ ) of the applied vibration and the impact (at $t=\tau$ ) of the primary mass on the base stopper. Then the response relative displacement of the secondary mass can be expressed by Duhamel's integral as [28]

$$
x_{s}(t)=\frac{1}{m_{s} \omega_{d}} \int_{0}^{t} p(\tau) e^{-\zeta_{s} \omega_{s}(t-\tau)} \sin \left[\omega_{d}(t-\tau)\right] d t
$$

where $p(\tau)$ is the arbitrarily varying excitation force at the time $\tau, \zeta_{s}=c_{s} / 2 \sqrt{k_{s} m_{s}}$ is the damping ratio, and $\omega_{d}=\omega_{s} \sqrt{1-\zeta^{2}}$ is the damped natural frequency in which $\omega_{s}$ is the resonant frequency of the secondary beam. Then the average stress on the surface of the secondary beam (piezoelectric bimorph) can be calculated as [29]

$$
\sigma_{a v g}=\frac{k_{s} X_{\max } l_{s} t_{e q}}{\left[\left(w t_{p}^{3} / 3\right)+4 w t_{p} t_{e q}^{2}\right]+E_{s h} w t_{s h}^{3} / 6 E_{p}}
$$

where $X_{\max }$ is the maximum displacement amplitude of the secondary mass, $w$ and $l_{s}$ are the width and effective length of the secondary beam, respectively; $t_{p}$ and $t_{s h}$ are the thicknesses of each piezo layer and middle shim layer of the bimorph beam, respectively while $t_{e q}=\left(t_{p}+t_{s h}\right) / 2$ is its equivalent thickness; $E_{p}$ and $E_{s h}$ are the Young's modulus of the piezo and shim materials, respectively. The output voltage generated and power delivered to a matched load resistance $R_{l}$ as a function of time $t$ can then be expressed as

$$
\begin{gathered}
V(t)=\frac{-d_{31} t_{p} \sigma_{a v g}}{2 \varepsilon_{r} \varepsilon_{0}} e^{-\xi_{s} \omega_{s} t} \sin \omega_{d} t \\
P(t)=\frac{1}{T} \int_{0}^{T} \frac{V(t)^{2}}{R_{l}} d t
\end{gathered}
$$

where $-d_{31}$ is the piezoelectric strain constant, $\varepsilon_{r}$ is the dielectric constant of the piezo material, $\varepsilon_{0}$ is the permittivity of free space, and $T$ is the time period of $V(t)$.

\subsection{Prototype fabrication}

A macroscale prototype energy harvester was fabricated to demonstrate the proof of concept. The primary springmass system which acts as the dynamic magnifier, is comprised of a flexible (polycarbonate) primary beam and a primary mass (Aluminum). The secondary spring-mass system which is the main energy harvesting (power generating) element is made of a rigid secondary beam (PZT bimorph) and a small secondary mass ( $\mathrm{NdFeB}$ cylinder), and is clamped on the primary mass. This double-beam structure is clamped to the harvester base (Aluminum) to form the EHDM (harvester without stopper). When a stopper is attached to the base (at approx. $1 \mathrm{~mm}$ distance from the fender of the primary mass), it forms the HSDM (harvester with stopper). Fig. 4 shows the photographs of both EHDM and HSDM mounted on the vibration exciter. The material properties and the dimensions of the PZT bimorph are given in Table 1. The primary mass is made larger than the secondary mass (mass ratio $\mu=5.8$ ) so that larger impulsive force could be 
Table 1. The material properties of PZT and dimension of the PZT bimorph

\begin{tabular}{c|c|c|c|c|c|c}
\hline Material properties & $\begin{array}{c}\text { Young's } \\
\text { modulus }\end{array}$ & $\begin{array}{c}\text { Piezoelectric strain } \\
\text { coefficient, }-d_{31}\end{array}$ & $\begin{array}{c}\text { Dielectric } \\
\text { constant }\end{array}$ & Density & Bimorph dimension & $\begin{array}{c}\text { Capacitance of the } \\
\text { bimorph }\end{array}$ \\
\hline PZT & $66 \mathrm{GPa}$ & $190 \mathrm{pC} \cdot \mathrm{N}^{-1}$ & 1730 & $7800 \mathrm{Kg} \cdot \mathrm{m}^{-3}$ & $40 \times 6 \times 0.6 \mathrm{~mm}$ & $19.7 \mathrm{nF}$ \\
\hline
\end{tabular}

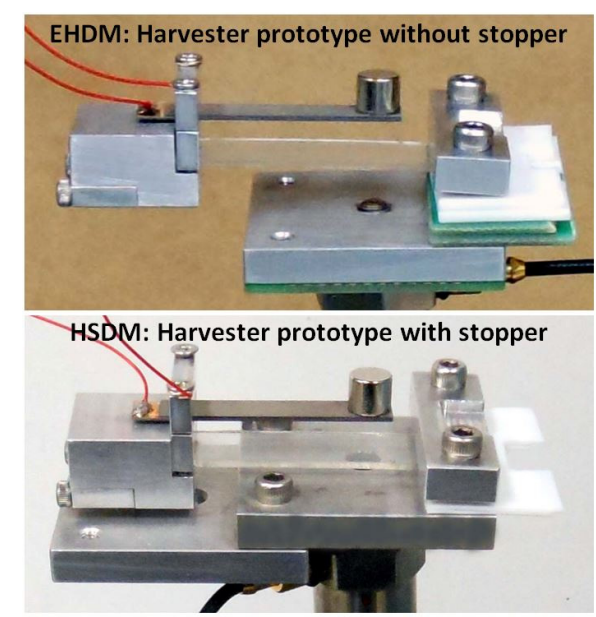

Fig. 4. Photographs of the fabricated energy harvester prototypes: EHDM (top) and HSDM (bottom)

Table 2. Dimensions of the double-beam structure used for both EHDM and HSDM

\begin{tabular}{c|c|c|c|c}
\hline Structure & \multicolumn{3}{|c|}{ Beam dimension (mm) } & \multirow{2}{*}{$\begin{array}{c}\text { Tip mass } \\
\text { (gram) }\end{array}$} \\
\hline & Length & Width & Thickness & \\
\cline { 1 - 4 } $\begin{array}{c}\text { Dynamic } \\
\text { magnifier }\end{array}$ & 32 & 11 & 1 & 6.1 \\
\hline $\begin{array}{c}\text { Harvester } \\
\text { element }\end{array}$ & 28 & 6 & 0.6 & 1.05 \\
\hline
\end{tabular}

generated during impact on the base stopper, even at low acceleration. Therefore, the secondary mass displacement increases which, in turn, enhances the strain on the piezoelectric surface, resulting in an increase in the output voltage and power. The geometric parameters of the double-beam structure are shown in Table 2.

\section{Experimental Results and Discussion}

\subsection{Experimental setup}

Fig. 5 illustrates the schematic diagram of the complete experimental setup along with the photograph of the harvester prototype under test (HSDM: harvester prototype with stopper). The fabricated prototype energy harvester was mounted on an electro-dynamic shaker (TMS 2004E) which is connected to a function generator (Agilent $33120 \mathrm{~A}$ ) in conjunction with a power amplifier (TMS 2004E21) in order to provide sinusoidal excitation of various frequencies and accelerations to the harvester prototype. The amplitude of the input vibration was monitored by a measuring amplifier (Type 2525 Brüel \&

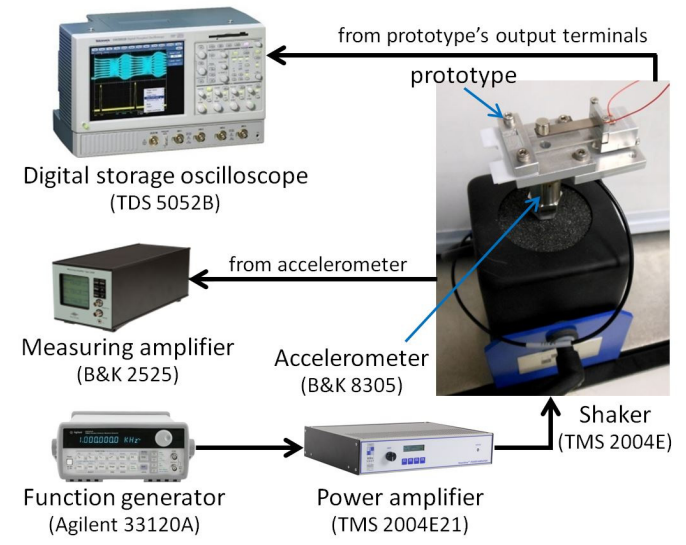

Fig. 5. Schematic diagram of the experimental setup with photograph of the HSDM prototype energy harvester under vibration exciter test

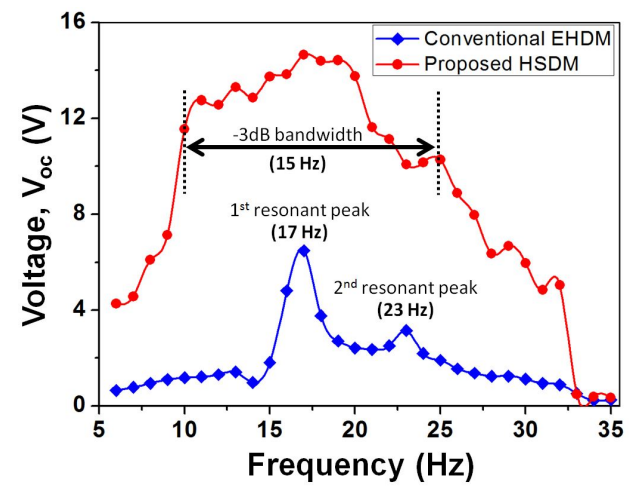

Fig. 6. Peak-peak open circuit voltage responses (under $1 \mathrm{~g}$ peak acceleration) of the HSDM in comparison with EHDM

Kjær) connected to a reference accelerometer (Type 8305 Brüel \& Kjær) that was attached to the base of the shaker. The output responses of the harvester were measured and recorded by a digital storage oscilloscope (Tektronix TDS5052B). In addition to the proposed HSDM, measurements were taken from the device without stopper that acts as a conventional EHDM in order to show the performance comparison.

\subsection{Results and discussion}

The frequency response of the peak-to-peak open circuit voltage of the HSDM along with the EHDM is shown in Fig. 6. It has been observed that the HSDM shows nonlinear frequency response with $15 \mathrm{~Hz}-3 d B$ bandwidth while, on the other hand, the EHDM shows a 2-DOF 


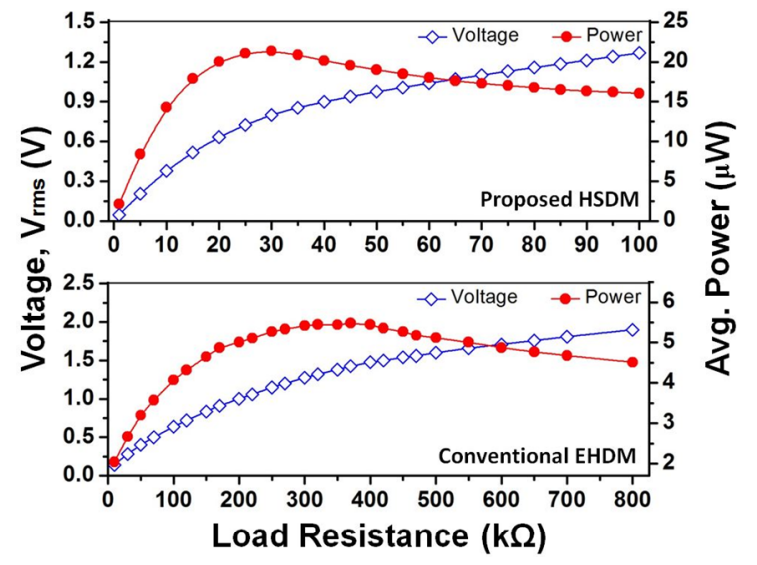

Fig. 7. Output voltage and power versus load resistance (at $17 \mathrm{~Hz}$ frequency and $1 \mathrm{~g}$ peak acceleration) obtained from the HSDM (top) and the EHDM (bottom)

frequency response with two consecutive resonant peaks at $17 \mathrm{~Hz}$ and $23 \mathrm{~Hz}$. When the primary mass impacts on the stopper, the effective stiffness of the primary beam changes, allowing the primary mass to deviate from its normal motion and enables the resonance to extend over a wider range. The peak-to-peak voltage is found to be more than double than that of the EHDM at the frequency of its 1 st resonance. An impulsive force during impact increases the stress on the PZT beam that allows increasing the voltage. Results show, maximum peak-peak open circuit voltages of $14.82 \mathrm{~V}$ and $6.94 \mathrm{~V}$ were generated by the HSDM and the EHDM, respectively at $17 \mathrm{~Hz}$ frequency and $1 \mathrm{~g}$ peak acceleration. The resultant maximum peak displacements of the secondary mass relative to the base (primary mass) of the secondary spring-mass system have been estimated as $590 \mu \mathrm{m}$ and $275 \mu \mathrm{m}$, respectively.

As shown in Fig. 7, the load voltages across various load resistances have been measured to determine the maximum power delivering capability of the proposed device. It was done at the 1st resonant frequency $(17 \mathrm{~Hz})$ of the EHDM for convenient comparison. The generated power is experimentally equal to $V_{r m s}^{2} / R_{l}$; where $V_{r m s}$ is the rms voltage across the load resistance $R_{l}$. Results show that the proposed HSDM delivers maximum $21.39 \mu \mathrm{W}$ average power to a $30 \mathrm{k} \Omega$ load resistance. On the other hand, the EHDM delivers $5.48 \mu \mathrm{W}$ average power to its optimal load resistance, $370 \mathrm{k} \Omega$. Mathematically, the value of optimal $R_{l}$ $\left(=R_{s}\right)$ can be determined by $[30]$

$$
R_{l}\left(=R_{s}\right) \propto \frac{1}{\omega_{n} C_{p}}
$$

where $R_{s}, \omega_{n}$, and $C_{p}$ are the source resistance, natural frequency, and capacitance of the piezoelectric beam, respectively. The frequency of the secondary (piezoelectric) beam is up-converted after the impact which, in turn, reduces the source resistance. Our proposed double-beam

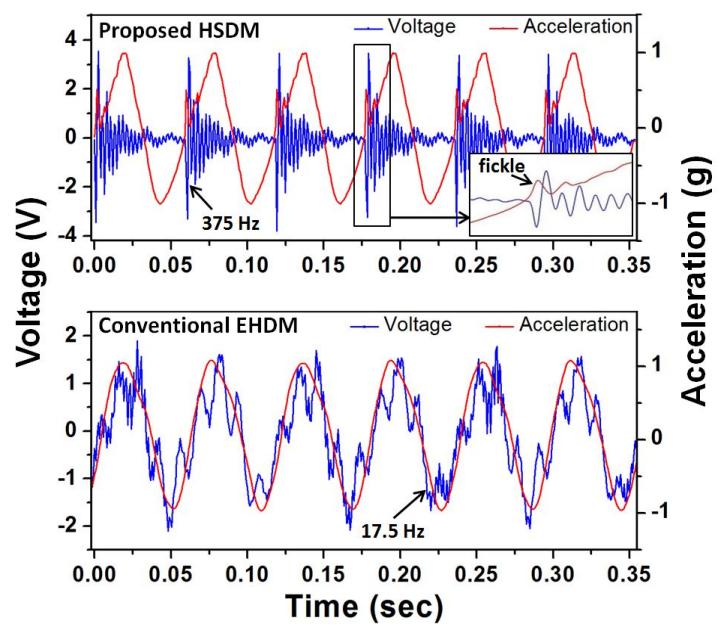

Fig. 8. Instantaneous voltage waveforms generated by the HSDM (top) and the EHDM (bottom) across the corresponding optimal load resistances at $17 \mathrm{~Hz}$ frequency and $1 \mathrm{~g}$ peak acceleration

system is a 2-DOF system having two close resonances. Therefore, the calculated value of the source resistance $R_{s}$ $\left(=R_{l}\right)$ may be affected by some dimensionless constant related to the natural frequencies of the proposed 2-DOF system.

Fig. 8 shows the instantaneously generated voltage waveform across the corresponding optimal load resistances of the HSDM and EHDM at $17 \mathrm{~Hz}$ frequency. The peak-topeak voltage of the HSDM right after an impact is quite high, as compared to the EHDM, but decays exponentially with time due to damping and becomes almost zero before the next impact occurs. As a result, the value of rms voltage $(0.8 \mathrm{~V})$ is very low than its peak-to-peak value $(7.35 \mathrm{~V})$. The up-converted frequency of the decaying waveform is $375 \mathrm{~Hz}$, obtained by Fast Fourier Transform (FFT). This fast exponential decay can be reduced by changing the characteristic (e.g. stiffness, damping coefficient) of the power generating element. It is to be noted that, part of the impulsive force generated by the impact between the primary mass and the harvester base is transferred to the shaker that results in fickle (shown inset) in the acceleration waveform. As seen, both the waveforms contain harmonics, resulting in the waving of the corresponding outputs. It happens because the flexible dynamic magnifier i.e., the primary spring-mass system, vibrates at low frequency with large displacement. As a result, vibration of the primary spring-mass system (that works as the base for the secondary spring-mass system) superimposes on the vibration of the secondary springmass system. Therefore, waving of the generated output voltage waveforms is a superposition of the mutual vibration of the two systems. The harmonic of EHDM introduces a slight change in the frequencies between its generated voltage waveform and the applied acceleration waveform. Also reduces the rms value of the generated 


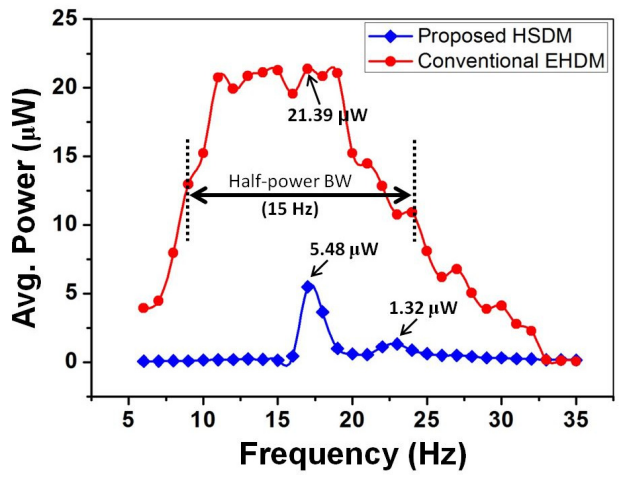

Fig. 9. Measured average powers delivered to respective optimum load resistances of the HSDM and the EHDM as a function of frequency under $1 \mathrm{~g}$ peak acceleration

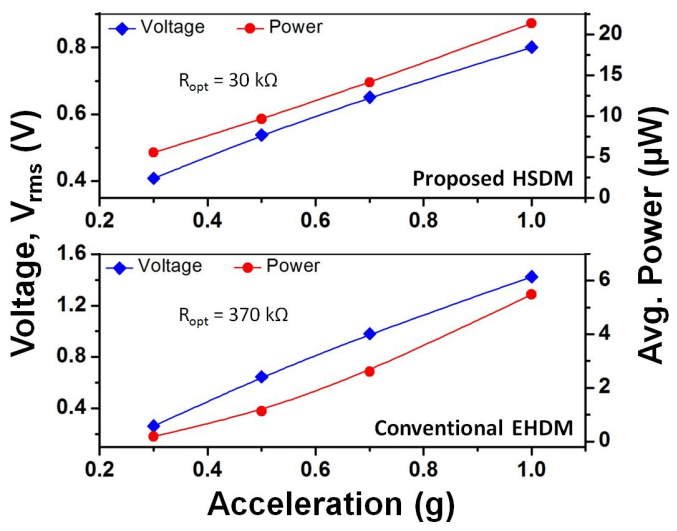

Fig. 10. Output voltage and power delivered to the corresponding optimum load resistances of the HSDM (top) and the EHDM (bottom) at $17 \mathrm{~Hz}$ under various base excitations

voltage than that obtained from a proper sinusoid.

Fig. 9 shows the average power delivered to the respective optimum load resistances as a function of the measuring frequency for both the devices. The EHDM generated maximum $5.48 \mu \mathrm{W}$ average power at its 1 st resonance $(17 \mathrm{~Hz})$ and $1.32 \mu \mathrm{W}$ average power at its 2 nd resonance $(23 \mathrm{~Hz})$. According to literature, its effective bandwidth is $6 \mathrm{~Hz}$ (from $17 \mathrm{~Hz}$ to $23 \mathrm{~Hz}$ ) [15]. On the other hand, the HSDM generated maximum $21.39 \mu \mathrm{W}$ average power with a half-power bandwidth of $15 \mathrm{~Hz}$ (from $9 \mathrm{~Hz}$ to $24 \mathrm{~Hz}$ ) which is $250 \%$ larger than that of its conventional counterpart, EHDM. Moreover, the average value of the average power from $11 \mathrm{~Hz}$ to $19 \mathrm{~Hz}$ was 18.4 $\mu \mathrm{W}$ that is almost four times higher than that of the EHDM at its 1 st resonance. The changes in output voltage and power delivered to the corresponding load resistances of the HSDM and the EHDM at $17 \mathrm{~Hz}$ excitation frequency (first resonance of the EHDM) with the change in base acceleration is shown in Fig. 10. Results show that both voltage and power increase linearly as the acceleration amplitude increases.
The experimentally obtained results show that the HSDM is a highly effective way of increasing both the operating bandwidth and the output power in low frequency vibration energy harvesting, specifically, in harvesting energy from automobile vibrations. Running engine in a stationary vehicle and a moving vehicle on the road produce low frequency vibrations. Depending on engine's rpm, vehicle speed, suspension system of the vehicle, road roughness etc., characteristics (frequency and amplitude) of generated vibrations are different. Moreover, occasional braking and speed-breakers on the road allow the vehicle to experience shock. The HSDM is applicable to both general vibration and shock vibration generated by vehicle. Use of flexible (polymer) beam material and mechanical stopper offers its reliable operation even when subjected to shock vibration. Further analysis and optimized design e.g., mass ratio, stopper distance, generating elements characteristics etc. and fabrication through MEMS process will improve its performance which are of our next interest.

\section{Conclusion}

In summary, a piezoelectric vibration energy harvester with stopper-engaged dynamic magnifier (HSDM) exhibits significantly improved performance in terms of both output power and wideband operation in low frequency energy harvesting, compared to a conventional energy harvester with dynamic magnifier (EHDM). Engagement of the base stopper with the mass of the flexible dynamic magnifier transforms an impulsive force generated by impact to the secondary power generating element, introducing a frequency up-conversion mechanism and non-linear motion of the magnifier's mass as well. Nonlinearity results in wideband operation and frequency upconversion allow the power to increase by increasing stress and decreasing source resistance in the generating element. As compared to its conventional counterpart, the device with stopper (HSDM) offers 250\% increased bandwidth $(15 \mathrm{~Hz})$ and almost four times increased average power $(21.39 \mu \mathrm{W})$. Optimization through further analysis and fabrication through MEMS process can further improve its performance.

\section{Acknowledgements}

This research was partially supported by a research grant of the Kwangwoon University in 2014, the Basic Science Research Program (2013R1A1A2A10064810), and the Pioneer Research Center Program (20100019313) through the National Research Foundation of Korea (NRF) funded by the Ministry of Science, ICT and Future Planning, Korea. The authors are grateful to the Micro/Nano Devices and Packaging Lab (MiNDaP) group members of Kwangwoon University. 


\section{References}

[1] Z. Xiao, T. Q. Yang, Y. Dong, and X. C. Wang, Energy harvester array using piezoelectric circular diaphragm for broadband vibration, Appl. Phys. Lett., Vol. 104, No. 22, pp. 223904-1-223904-4, Jun. 2014.

[2] E. K. Reilly, F. Burghardt, R. Fain, and P. Wright, Powering a wireless sensor node with a vibrationdriven piezoelectric energy harvester, Smart Mater. Struct., Vol. 20, No. 12, pp. 125006, Nov. 2011.

[3] A. Mathers, K. S. Moon, and J. Yi, A vibration-based PMN-PT energy harvester, IEEE Sens. J., Vol. 9, No. 7, pp. 731-739, Jul. 2009.

[4] S. R. Anton and H. A. Sodano, A review of power harvesting using piezoelectric materials (2003-2006), Smart Mater. Struct., Vol. 16, No. 3, pp. R1-R21, May 2007.

[5] C. B. Williams and R. B. Yates, Analysis of a microelectric generator for micro systems, Sens. Actuators A, Vol. 52, No. 1-3, pp. 8-11, Mar.-Apr. 1996.

[6] H. Külah and K. Najafi, Energy scavenging from low-frequency vibrations by using frequency upconversion for wireless sensor applications, IEEE Sens. J., Vol. 8, No. 3 pp. 261-268, Mar. 2008.

[7] A. F. Arrieta, P. Hagedorn, A. Erturk, and D. J. Inman, A piezoelectric bistable plate for nonlinear broadband energy harvesting, Appl. Phys. Lett., Vol. 97, No. 10, pp. 104102-1-104102-3, Sep. 2010.

[8] S. Qi, R. Shuttleworth, S. O. Oyadiji, and J. Wright, Design of a multi-resonant beam for broadband piezoelectric energy harvesting, Smart Mater. Struct., Vol. 19, No. 9, pp. 094009, Aug. 2010.

[9] H. Xue, Y. Hu, and Q. -M. Wang, Broadband piezoelectric energy harvesting devices using multiple bimorphs with different operating frequencies, IEEE Trans. Ultrason. Ferroelectr. Freq. Control, Vol. 55, No. 9, pp. 2104-2108, Sep. 2008.

[10] Z. Yang and J. Yang, Connected vibrating piezoelectric bimorph beams as a wide-band piezoelectric power harvester, J. Intell. Mater. Syst. Struct., Vol. 20, No. 5, pp. 569-574, Jan. 2009.

[11] H. Liu, C. Lee, T. Kobayashi, C. J. Tay, and C. Quan, Investigation of a MEMS piezoelectric energy harvester system with a frequency-widened-bandwidth mechanism introduced by mechanical stoppers, Smart Mater. Struct., Vol. 21, No. 3, pp. 035005, Feb. 2012.

[12] X. Tang and L. Zuo, Enhanced vibration energy harvesting using dual-mass systems, J. Sound Vib., Vol. 330, No. 21, pp. 5199-5209, Jun. 2011.

[13] Q. Ou, X. Chen, S. Gustschmidt, A. Wood, N. Leigh, and A. F. Arrieta, An experimentally validated double-mass piezoelectric cantilever model for broadband vibration-based energy harvesting, $J$. Intell. Mater. Syst. Struct., Vol. 23, No. 2, pp. 117-126, Jan. 2012.

[14] H. Wu, L. Tang, Y. Yang, and C. K. Soh, A novel two- degrees-of-freedom piezoelectric energy harvester, $J$. Intell. Mater. Syst. Struct., Vol. 24, No. 3, pp. 357368, Jan. 2013.

[15] O. Aldraihem and A. Baz, Energy harvester with a dynamic magnifier, J. Intell. Mater. Syst. Struct., Vol. 22, No. 6, pp. 521-530, May. 2011.

[16] W. Zhou, G. R. Penamalli, and L. Zou, An efficient vibration energy harvester with a multi-mode dynamic magnifier, Smart Mater. Struct., Vol. 21, No. 1, pp. 015014, Jan. 2012.

[17] A. Aladwani, M. Arafa, O. Aldraihem, and A. Baz, Cantilevered piezoelectric energy harvester with a dynamic magnifier, J. Vib. Acoust., Vol. 134, No. 3, pp. 031004, Apr. 2012.

[18] O. Zorlu, E. T. Topal, H. and Kulah, A vibration-based electromagnetic energy harvester using mechanical frequency up-conversion method, IEEE Sens. J., Vol. 11, No. 2, pp. 481-488, Feb. 2011.

[19] H. Liu, C. Lee, T. Kobayashi, C. J. Tay, and C. Quan, Piezoelectric MEMS-based wideband energy harvesting systems using a frequency-up-conversion cantilever stopper, Sens. Actuators A, Vol. 186, pp. 242-248, Feb. 2012.

[20] M. A. Halim and J. Y. Park, Theoretical modeling and analysis of mechanical impact driven and frequency up-converted piezoelectric energy harvester for lowfrequency and wide-bandwidth operation, Sens. Actuators A, Vol. 208, pp. 56-65, Jan. 2014.

[21] I. Sari, T. Balkan, and H. Kulah, An electromagnetic micro power generator for low-frequency environmental vibrations based on the frequency up conversion technique, J. Microelectromech. Syst., Vol. 19, No. 1, pp. 14-27, Feb. 2010.

[22] Q. C. Tang, Y. L. Yang, and X. Li, Bi-stable frequency up-conversion piezoelectric energy harvester driven by non-contact magnetic repulsion, Smart Mater. Struct., Vol. 20, No. 12, pp. 125011, Nov. 2011.

[23] P. Pillatsch, E. M. Yeatman, and A. S. Holmes, A piezoelectric frequency up-converting energy harvester with rotating proof mass for human body applications, Sens. Actuators A, Vol. 206, No. 1, pp. 178-185, Feb. 2014.

[24] M. A. Halim and J. Y. Park, Performance enhancement of a low frequency vibration driven 2-DOF piezoelectric energy harvester by mechanical impact, in Proc. PowerMEMS, 2014.

[25] S. W. Shaw and P. J. Holmes, A periodically forced piecewise linear oscillator, J. Sound Vib., Vol. 90, No. 1, pp. 129-155, Sep. 1983.

[26] S. W. Shaw, Forced vibrations of a beam with onesided amplitude constraint: Theory and experiment, $J$. Sound Vib., Vol. 99, No. 2, pp. 199-212, Mar. 1985.

[27] L. Tang and Y. Yang, A multiple-degree-of-freedom piezoelectric energy harvesting model, J. Intell. Mater. Syst. Struct., Vol. 23, No. 4, pp. 1631-1647, Jun. 2012. 
[28] C. Lalanne, Mechanical Vibration and Shock Analysis-volume 2, John Wiley \& Sons, pp. 51-94, Jan. 2010.

[29] L. Gu, Low frequency piezoelectric energy harvesting prototype suitable for the MEMS implementation, Microelectr. J., Vol. 42, No. 2, pp. 277282, Feb. 2011.

[30] S. Adhikari, M. I. Friswell, and D. J. Inman, Piezoelectric energy harvesting from broadband random vibrations, Smart Mater. Struct., Vol. 18, No. 11, pp. 115005, Sep. 2011.

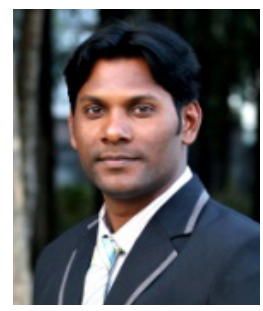

Miah Abdul Halim He received his B.Sc and M.Sc in Applied Physics Electronics and Communication Engineering from Islamic University, Bangladesh, in 2007 and 2008, respectively. He worked as a research student in the Experimental Physics Division at Atomic Energy Centre Dhaka (AECD), Bangladesh. His M.Sc thesis was on Synthesis and Characterization of Zinc Selenide ( $\mathrm{ZnSe}$ ) Thin Films. He is currently a $\mathrm{PhD}$ student in the Department of Electronic Engineering at Kwangwoon University, Seoul, Korea. He is pursuing his $\mathrm{PhD}$ in human motion based energy harvesting devices and systems since March'12 under supervision of Dr. Jae Yeong Park. His main research interests include harvesting energy from human-body-induced vibration, shock-driven energy harvester for automobiles application, and high frequency MEMS ultrasonic devices for biomedical imaging.

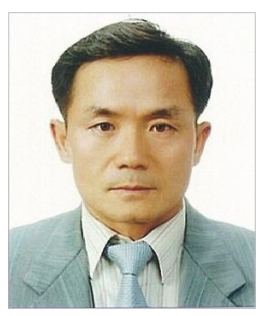

Dae Heum Kim received his B.Sc degree in Chemical Technology from Seoul National University, Korea in 1982, M.Sc and Ph.D degrees in Polymer Science, from University of Akron, Ohio, U.S.A. in 1987 and 1990. After graduation, he has been working as a professor in Department of Chemical Engineering in Kwangwoon University. His research interests include Electrically conducting polymer composites with nano particles, and Artificial Muscle and Vein.

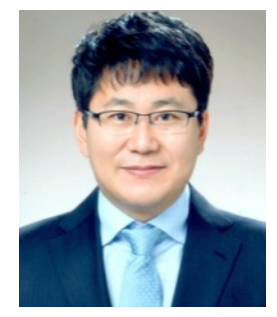

Jae Yeong Park He received his Ph.D. degree in electrical and computer engineering from Georgia Institute of Technology, Atlanta, in 1997. After graduation, he worked as a research scientist at the same institution for more than one year. Then he joined as a Senior Research Engineer in LG Electronic Institute of Technology, Korea in 1999 and promoted as Chief Research Engineer in 2001. In September 2004, he joined as an Assistant Professor in the Department of Electronic Engineering of Kwangwoon University, Korea and presently he is working as a Professor. He has contributed to more than 200 international conference papers and peer-reviewed journal articles in the fields of piezoelectric MEMS sensors and actuators, Optical MEMS, RF MEMS, Bio-sensors, and Energy harvesting. He is holding more than 110 patents on his name. He is also a reviewer of a number of renowned journals in the fields mentioned above. His current research interests include Low-vibration-based MEMS energy harvesting devices and systems, Piezoelectric micro-actuators for optical and RF applications, High frequency MEMS ultrasonic devices for biomedical imaging, and printed circuit board embedding passive devices and chip technology. 